\title{
Césure et sublime ${ }^{1}$
}

1. Après tant d'années, j'ai relu le premier roman de Kundera, „La Plaisanterie” et j'ai été frappé par la force de son expression, et par, je dirais, son volume, sa masse romanesque. J'entends la densité des phrases, leur énergie; la multitude des situations décrites; la quantité même des mots - en comptant en général et par page - sans beaucoup d'alinéas, "La Plaisanterie” étant le plus long des romans kunderiens, plus long que „L'Insoutenable légèreté de l'être” et „L'Immortalité”, si on prenait en considération le nombre de signes. C'est aussi le roman kunderien le plus apprecié par le public intello dans mon pays; ou que je parle à l'université de Kundera, on l'évoque en premier lieu - bien que le roman qui s'était le mieux vendu auprès du grand public fût „L'Insoutenable légèreté de l'être”.

Bien évidemment, répondre à la question journalistique "quel est pour vous le meilleur livre de Kundera" - et en tant que son traducteur, j'entends souvent me la poser - n'a aucun sens.

J'ai été impresionné par la relecture de "La Plaisanterie”, mais pour une autre raison que la qualité littéraire - évidente et absolument époustoufflante - du roman. J'ai vu un plus clairement le chemin esthétique emprunté par Kundera le romancier et qui l'a conduit du premier à son dernier roman, "la Fête de l'insignifiance”.

Un des clichés qui accompagne la réflexion critique sur l'oeuvre de Kunder est „la césure” qui séparerait celle-ci en deux parties. Dans la plupart des cas, on oppose la période tchèque à la période française, les romans écrits en tchèque aux romans écrits en français, éventuellement les romans dont l'action se déroule en Tchécoslovaquie (ou en Tchéquie) aux romans ancrés dans la réalité occidentale, française en l'occurence.

1 Pierowdruk: „Atelier du roman” mars 2020, no 100. 
On peut facilement dessiner de telles lignes de partage, c'est clair, mais à mon sens elles n'ont pas de grande valeur sauf statistique. À la place je proposerais une autre approche de l'évolution de l'oeuvre de Kundera. Je situerais la césure, s'il y en a une, très tôt, notamment après „La Plaisanterie”. Non seulement le premier roman de Kundera mais aussi le seul où il n'y a aucune narration extérieure, où la narration est consituée des monologues des protagonistes; la seule où le narrateur est totalement absent, n'intervient pas, n'existe pas comme dans les autres romans, y serait-il invisible ou ostentoirement présent. Dans „La Plaisanterie”, il n'est même pas un deus absconditus; il n'est pas invité dans ce roman, ses fonctions ayant été cédées aux personnages romanesques.

Le procédé se révélera unique. C'est un procédé formel, il faut dire, puissant et efficace, si l'on sait s'y mettre. Assez rarement utilisé, moins souvent que son cousin dans l'histoire du roman: échange des lettres dans le roman dit épistolaire. Mais il ne faut pas oublier qu'il a un aìeul important: „Le Décameron”. Le dernier exemple le plus réussi de cette démarche, qui me vient à la tête, est le chef d'oeuvre de Roberto Bolaño „Les détectives sauvages" (1998).

Le danger de ce procédé où le narrateur, cédant la parole aux personnages, se lave les mains, tel Pilate, consiste à en faire trop: comme chaque personnage "devient" responsable de son histoire à raconter, on risque d'entendre plus qu'il ne faudrait; les propos s'allongent, le contrôle qu'aurait exercé le narrateur (invisible ou présent) se perd, les mots passent plus facilement. Chez Bolaño il y en a vraiment beaucoup; une telle prolixité serait impensable chez Kundera. Cependant, cette manière de construire le roman rend moins visible, et par moments carrément élimine la figure de style qui en même temps constitue chez Kundera le moyen de connaissance romanesque et qui sera ensuite sa spécialité de la maison: l'ellipse.

Dans les romans suivants les personnages ne remplacent donc plus le narrateur et ne font plus de longs monologues à la première personne; la présence du narrateur se fait sentir de plus en plus jusqu'à faire de lui un personnage à part entière, présent dans le livre charnellement (comme dans "I'Immortalité" ou „La Lenteur") ou par défaut (comme le Maître dans la "Fête de l'insignifiance"). En même temps la densité et la longuer des propos proférés par les protagonistes se raréfient; nous passons à un autre registre du romanesque. „La Plaisanterie” cédera place aux romans plus dépouillés d'abord, et presque ascètes ensuite, construits sur d'autres principes qui seront plus fidèles à l'art du roman tel que Kundera conçoit au fur et à mesure que son oeuvre progresse.

Il y a tout au long de l'oeuvre kundérienne comme un mouvement qui part de ce qui est massif, lourd (au sens du poids physique: quantité de mots), vers ce qui est allégé, transparent. Le dernier livre, „La Fête de l'insignifiance", le roman le plus court, le plus désincarné, pour ainsi dire, clora ce processus; le corps du roman se retrécira jusqu'au minimum, et une pensée libre, sans emploi, sans explication, sans racines, bref une pensée indéterminée s'en dégagera. C'est pourquoi je conçois la voie de cette création comme un passage interne (chaque artiste dessine sa propre voie, un trajet 
pour son oeuvre qui est souvent mal compris ou invisible de l'extérieur) du plein au vide.

Ce vide que j'appelle la pensée indéterminée et libre a quelque chose de la gnosis; une pensée s'échappe à la fin de la forme romanesque, de la matière romanesque, du corps du roman (faut-il y voir aussi comme un écho de l'enfantophobie qui touche plus d'un des personnages romanesques kunderiens?). Le processus, s'il en est un, serait parallèle à la voie parcourue par ceux parmi les personnages kunderiens qui semblent les plus intelligents, les plus avertis, les plus lucides: aussi bien Agnès que Sabine (et d'autres encore) se libèrent dans la mesure du possible du poids de leur existence pour en arriver à l'état où entre leur âme et le monde tel qu'il est il y aurait le moins d'attaches possible.

On ne peut pas s'empêcher de penser au dernier roman de Flaubert, „Bouvard et Pécuchet". Et plus exactement à son deuxième volume, jamais terminé, connu sous le titre "Copie" (tandis que le premier volume avait pour le titre provisoire „Le Roman”). Il allait être rempli presque exclusivement des citations copiées par les deux „bonhommes” des différents livres. Il en resulterait - on serait enclin à croire - une démistification complète de la langue, y compris du langage romanesque pratiqué par Flaubert. Kundera rêvant depuis longtemps au "roman où aucun mot ne serait sérieux" refait à sa manière le geste flaubertien: se détacher de sa propre création, déjouer ses propres mécanismes, faire un roman qui n'en serait pas un et qui pourtant serait considéré comme tel, écrire un roman "bête" au sens flaubertien, c'est-à-dire un roman qui serait conscient de są propre "bêtise”.

J'ai toujous devant mes yeux la scène d'une très ancienne émission télévisée (encore en blanc et noir) où Kundera, le quadragénaire au regard d'un ange pervers, parlant de son premier roman dans un français approximatif et se balançant dans un fauteuil idiot - mou. en forme de ballon - répète: „La plaisanterie est une catégorie fondamentale pour saisir notre être". Plus de quarante ans après, il dit à peu près la même chose dans son dernier roman: l'insignifiance est une catégorie fondamentale...etc. Le cercle de la spirale revient au point de départ, la boucle semble bouclée. Sauf que la plaisanterie d'autrefois révélait la vérité de l'existence soumise directement aux pressions (et à l'oppression) de l'Histoire, tandis que l'insignifiance serait aussi une catégorie esthétique qui déterminerait la création artistique au bout du chemin parcouru par le romancier. Elle enlèverait au roman le sérieux de sa vocation (que Kundera, dans la lignée de Broch, a toujours défendu). Elle mettrait entre les parenthèses le dessein épistémologique du roman, et elle changerait le livre en une fête ou aucune précision, aucune exigence formelle et éthique (tout ce qui appartient à l'éthique, au devoir du roman) ne sont plus requises.

2. L'insignifiance est aussi une forme de sublime. Je l'ai compris tardivemenet, justement à la lumière de la relecture de „La Plaisanterie”.

En décrivant la dette française envers Kundera Alain Finkielkraut dit dans une interivew que celui-ci a délivré le lecteur (et l'écrivain) français de l'avant-garde; „ou plus exactement il a délivré la modernité de sa captation 
par l'avant-garde". Pour le lecteur centre-européen l'essentiel de l'apport kunderien était l'esthétisation (et par conséquant, "existentialisation") de notre rapport envers l'Histoire. Nous dire en 1984 que notre ennemi n'était pas le communisme (comme l'émanation actuelle de l'Histoire), mais le kitsch, était un défi révolutionnaire, diffcilement acceptable uand on voyait des chars militaires dans le rues. Aujourd'hui on ne peut que voir encore mieux le bien-fondé de cette approche: si Kundera nous a très bien parlé de notre actualité politique de l'époque, il a encore mieux parlé de notre futur qui allait advenir après la chute du communisme et ouvrir la voie à une autre émanation de l'Histoire que sera le libéralisme postcommuniste, et plus tard le nationalisme postcommuniste, toutes ces émanations étant de puissantes usines du kitch.

Les différences entre la lecture „occidentale” et la lecture „centre-européenne" s'estompent cependant ou passent au deuxième plan, quand on admet qu'il y a une catégorie supérieure commune pour les deux lectures: celle, mentionnée à l'instant, du sublime.

Si les premiers romans de Kundera, de „La Plaisanterie” jusqu'à „L'Insoutenable légèreté de l'être" ont eu le plus de retentissement et ont provoqué le plus d'enthousiasme, c'est aussi parce que ces romans recelaient plus directement - et je dirais, plus facilement - le sentiment de sublime. Ce sublime résulte de la rencontre - si typiquement kunderienne - entre la grande Histoire et l'histoire individuelle des protagonistes. Les protagonistes kunderiens menaient leurs existence intime, découvraient ses différentes formes face à la grande Histoire, en l'occurrence l'oppression communiste qui faisait sentir sa présence sans répit, tous les jours. Une fois plus, d'autres fois moins fort, mais toujours elle planait en l'air, était quelque part là. Cette Histoire était une grandeur inconcevable, incompréhensible, hors de la portée des individus. Dans la définition kantienne du sublime elle jouerait justement le rôle de la puissance auquelle l'homme n'a pas d'accès, ce qui provoque chez lui le sentiment de crainte, et par conséquant aussi du sublime: le sublime naît là où une réalité énorme nous dépasse, devient insaisissable et nous restons devant elle comme prosternés. Mais en même temps le sublime apporte un certain goût à l'existence, une tension énergisante, une exaltation qui confère au quotidien comme une sorte de fièvre; comme dit Kant, "nous y prenons plaisir mêlé d'effroi". Le lecteur occidental, même si certaines côtés et nuances de la vie quotidienne sous le communisme lui échappaient, ressentait ce sublime d'autant qu'il faisait défaut dans les romans occidentaux et dans la réalité dans laquelle il vivait. Et le sublime ressenti dans la littérature est peut-être quelque chose d'absolument fondamental - et si rarement présent dans la création romanesque d'ajourd'hui.

Avec „L'Immortalité” le sublime devenait beaucoup moins palpable. La grande Histoire était bien là, mais sous la forme de l'imagologie et non pas sous la forme de répression, de menaces, des choses pour ainsi dire définitives, plus violentes, moins floues. Et cette forme-là, j'entends imagologie (aujourd'hui nous dirions aussi le turbo-capitalisme ou le néolibéralisme), n'étant pas alors une force sufisamment puissante, suffisamment obscure, suffisamment étrangère, le lecteur occidental n'arrivait pas à reprendre la 
même lecture que celle des romans précédants. Et cependant les événements des dernières années ont démontré que cette force-là s'est nettement affermie et est devenue aussi puissante et aussi menacante que ne l'était l'Histoire tissée par les idéologies et leur régimes policiers.

Dans le dernier roman de Kundera avec "l'insignifiance" on découvre encore une autre dimension de cette "grandeur" du monde à laquelle nous devons nous affronter, en face de laquelle nous devons nous identifier. L'insignifiance se situe en dehors de l'histoire totalitaire opressive, en dehors du marché libre monothéiste oppressif ou plutôt tout en les englobant elle les dépasse; elle est comme une réalité encore plus „profonde” et plus large, plus étendue, elle constitue le fond de toile complet, cette "mer" infinie du fameux tableau de Friedrich devant laquelle l'homme sent sa nullité et en même temps un frisson d'être.

Face à cette insignifiance universelle, il ne nous reste probablement que la bonne humeur. Un état chez Kundera non pas psychologique mais métaphysique: frisson de sens dans le monde insignifiant.

\section{SUMMARY}

Having written The Joke, Kundera changed the modus vivendi of the novel's narrator who, with time, became a separate protagonist. This is connected with the appearance of the ellipsis as the main plot device, which is not yet present in The Joke. This change in the way of narrating represents another and, perhaps, a deeper dividing line in Kundera's writing than switching to another language (from Czech to French), the change of the setting (from Czechoslovakia to Western countries) and the change of the political system (from communist to 'imagological') as an instrument of oppression.

\section{Keywords}

Kundera, novel, narrator, sublimeness, story, ellipsis

Marek Bieńczyk - professor in the Institute of Literary Research if the Polish Academy of Sciences. He wrote many essays devoted to world literature and studies on melancholy and Polish Romanticism. He is also a prose writer and a translator. He received the Nike Prize in 2012.

e-mail: marek.bienczyk@ibl.waw.pl 